\title{
Effectiveness of Antivirals and Macrolide or Tetracycline Antibiotic therapy for Feline Peritonitis Virus
}

\author{
By Pardis Tabaee Damavandi
}

\section{Introduction}

Feline Peritonitis Virus (FIP) is the main complication to Feline Coronavirus which is a result of its mutation "in vivo" and can be transmitted across large populations of felidae (Feles cati) via bodily fluids, fur grooming and litter condivision. The exacerbation of the disease can lead to either effusive FIP or the non-effusive forms which can both be fatal. Among the symptoms, difficulty breathing, lethargia, loss of appetite, vomiting/diarrhea, jaundice, neurological disorders, such as nystagmus and ataxia can be observed resulting in poor quality of life. FIP affects both young and adult cats with the younger population being at exceptionally high risk. In $80 \%$ of the cats the disease is solved with mild symptoms in around a week with excretion of the virus through elimination, however, in some cases further challenges may arise due to the virus being active and able to infect immunocompromised cats.

\section{Therapy options for FIP}

Current therapy consists of tetracycline antibiotics, such as Doxycycline which may be successful due to its access and ability to interact with the viral RNA and macrolide antibiotics such as Erythromycin again due to its similar effects. These two classes of antibiotics are wide spectrum and can their effects extend further towards both proteic and ribonucleic inhibition being that they are designed to target ribosomial substrates that present a hybrid composition of both proteic and ribonucleic structures. These drugs are howbeit expensive and therapy duration can be of months with resolution of the disease only detectable 5-9 months into therapy. This is due to their aspecificity. 


\section{Discussion}

Neverthless, the quick analysis of the Feline peritonitis virus structure, obtained with sequence analysis methodology, reveals high homology with both the Feline Coronavirus and the human Coronavirus. Like the human forms, the Glycine-Valine (GV) sequences are featured and set off the MAP kinase cascade particularly at gastroenteric and respiratory level. The GV scaffolds are numerous as well as the multiple Histidine $(\mathrm{HHHHH})$ sequences, making the virus an ideal target to Antiviral therapy or vaccination, however this strategy needs to be further scrutinised by veterinarians to confirm that Glycine-Valine sequencing is not otherwise found in the cat's native proteins, hormones, enzymes, or any other endogenous feline proteic based structures. 